\title{
High Fibre Diet Reduced the Energy Cost of Production and Abdominal Fat of Broiler Chickens
}

\author{
Md. Momenuzzaman Bhuiyan ${ }^{1 *}$, Zhijun Cheng ${ }^{1}$, Md. Saiful Bari ${ }^{1,2,3}$, Paul A. Ij ${ }^{1,4}$
}

${ }^{1}$ School of environmental and Rural Science, University of New England, Armidale, NSW 2351, Australia; ${ }^{2}$ Department of Dairy and Poultry Science, Chattogram Veterinary and Animal Sciences University, Khulshi, Chattogram 4225, Bangladesh; ${ }^{3}$ CSIRO, Agriculture and Food, New England Highway, Armidale, NSW 2350, Australia; ${ }^{4}$ Dean, College of Agricultural, Fisheries and Forestry, Fiji National University, Fiji.

\begin{abstract}
High fibre diet might reduce feed cost of broilers but increase the meat quality. We investigated the meat quality and feed cost by the inclusion of high fibre in broiler chickens' diets. Two hundred and eighty-eight day-old Cobb 500 broiler chickens, (about $42 \mathrm{~g}$ each), were fed on the 6 treatments, each replicated 6 times, with 8 birds per replicate. The chicks were fed on commercial-types diets from 1-10 days (starter). Subsequently, they were offered grower (11-24 days) and finisher (25-35 days) diets, which differed in fibre and energy contents. The diets were similar in nutrient composition, formulated in line with Cobb requirements. The chicks were reared in cages in a climatecontrolled room with ad libitum water and feed. Feed intake (FI), body weight gain (BWG), and feed conversion ratio (FCR) were measured. At day 35, visceral organs, breast, thigh, drumstick and fat pad were collected and weighed. At the same day, one more bird was killed (after 4-hour fasting) through cervical dislocation and a subsample of the whole bird was taken after chopping for fat analyses. Birds on the high-fibre diets did not differ significantly in weight gain but were more efficient in nutrient utilization. High-fibre diets stimulated the development of the gastrointestinal tract, with heavier intestines $(\mathrm{P}<0.006)$, and proventriculus $(\mathrm{P}<0.026)$ at 24 days, and small intestine $(\mathrm{P}<0.006)$ and liver $(\mathrm{P}<0.020)$ at 35 days. Dietary fibre content significantly lowered $(\mathrm{P}<0.002)$ abdominal fat pad, and increased $(\mathrm{P}<0.010)$ breast meat yield. Feed cost was reduced due to a reduction in energy content in the grower ( $\$ 0.54$ vs 0.58 per bird) and finisher ( $\$ 0.54$ vs 0.57 per bird) phases. Low dietary energy combined with cheap high-fibre ingredients greatly reduced the costs of diets and reduce the carcass fat content.
\end{abstract}

Keywords | Broiler, Cobb 500, Carcass, Feed cost, Lipid, Meat quality

Received | January 01, 2021; Accepted | July 01, 2021; Published | August 15, 2021

*Correspondence | Md. Momenuzzaman Bhuiyan, ${ }^{1}$ School of environmental and Rural Science, University of New England, Armidale, NSW 2351, Australia; Email: mbhuiya4@une.edu.au

Citation | Bhuiyan M, Cheng Z, Bari MS, Iji PA (2021). High fibre diet reduced the energy cost of production and abdominal fat of broiler chickens. Adv. Anim. Vet. Sci. 9(10): 1585-1593.

DOI | http://dx.doi.org/10.17582/journal.aavs/2021/9.10.1585.1593

ISSN (Online) | 2307-8316; ISSN (Print) | 2309-3331

Copyright $\odot 2021$ Bhuiyan et al. This is an open access article distributed under the Creative Commons Attribution License, which permits unrestricted use, distribution, and reproduction in any medium, provided the original work is properly cited.

\section{INTRODUCTION}

$\mathrm{F}$ eed is one of the most important factors which impacts broiler performance (Ferket and Gernat, 2006; Dozier et al., 2010). Broilers consume a large amount of feed. Feed costs vary with the cost of ingredients but normally feed costs for broilers represent $65-85 \%$ of the variable cost of production of the live broiler (Parkhurst and Mountney, 1988; da Costa et al., 2017). This cost has risen significantly in the past few years due to increases in prices of many feed ingredients, particularly cereal grains, which are used mainly as a source of energy for the birds (Donohue and Cunningham, 2009). Total feed costs can be reduced by the inclusion of less grain in the diet. Such a practice would also improve the quality of poultry meat, which generally becomes fatty as a result of the very rapid growth of birds and over-consumption of very high-quality feed.

Several studies have indicated that lowering dietary energy can reduce fat deposition in broiler carcass (Griffiths et 
al., 1977; Mabray and Waldroup, 1981; Mcleod, 1982; Khantaprab et al., 1997; Lippens et al., 2000). The reduction of cereals, especially wheat, content in the diets can reduce dietary energy.

Enzymes can then be used to improve the low energy diet (Zhou et al., 2009; Saleh et al., 2018). The wheat-based diet contains a high level of non-starch polysaccharides, the enzyme can increase digestibility due to degradation of non-starch polysaccharide.

Dietary fibre is traditionally considered as diet diluents and often, an anti-nutritional factor (Kras et al., 2013). However, moderate amounts of fibre may promote organ development, nutrient digestibility and enzyme production in poultry (Mateos et al., 2012). Some of these effects are caused by better gizzard function (Gonzalez-Alvarado et al., 2007), with an augmentation in the gastroduodenal reflux that promotes the contact between digestive enzymes and nutrients (Kras et al., 2013; Jorgensen et al., 1996).

Cellulose has been included in poultry diets as a source of dietary fibre (Mohiti-Asli et al., 2012). Mohiti-Asli et al. (2012) reported that dietary cellulose affected reducing abdominal fat deposition. Moreover, the authors (MohitiAsli et al.,2012) pointed out that dietary cellulose depressed lipid accumulation in the liver of growing broilers under $a d$ libitum feeding.

Considering the above facts, we conducted the study with the aim to test the benefits of high-fibre diets on feed cost and abdominal meat quality of the broiler chickens.

\section{MATERIALS AND METHODS}

\section{BIRDS HOUSING AND FEED MANAGEMENT}

This was a 3 X 6 dietary factorial study with 3 different fibre content and 6 energy levels (Table 1). Each treatment was replicated 6 times with 8 chicks per replicate.

Table 1: The experimental diets arrangement including dietary fibre content and energy level for starter, grower and finisher phases.

\begin{tabular}{|c|c|c|c|c|}
\hline Fibre content (\%) & 2.5 & & 3.5 & 4.5 \\
\hline \multirow{2}{*}{$\begin{array}{l}\text { Energy Level } \\
(\mathrm{MJ} / \mathrm{kg})\end{array}$} & 13.0 & 12.5 & $13.0 \quad 12.5$ & 13.0 \\
\hline & $\begin{array}{l}\text { Positive } \\
\text { control }\end{array}$ & $\begin{array}{l}\text { Negative } \\
\text { control }\end{array}$ & Test diets & Test diets \\
\hline
\end{tabular}

Two hundred and eighty eight day old Cobb 500 broiler chickens, (about $42 \mathrm{~g}$ each), were fed on the 36 cages with 8 birds in each cage. When the chickens were placed into the cages, the water supply and feed trough were adjusted to fit their height.
The initial environment temperature in the house was $33^{\circ} \mathrm{C}$. Afterwards, the environment temperature was gradually reduced to $22^{\circ} \mathrm{C}$ on day 21 . From 21 to 35 days, the temperature was set at $21-22^{\circ} \mathrm{C}$.

Feed and water were provided ad libitum. Chickens were weighed on day 1, and again on days 10, 24 and 35 . Leftover feed was weighed at the same time on days 10,24 and 35. Mortality was recorded as it occurred. On day 24, two chickens from each cage were weighed and slaughtered by cervical dislocation and their organs (gizzard, small intestine, proventriculus, pancreas, liver, bursa and spleen) were weighed too.

Table 2: Diet compositions for starter birds (0-10 days).

\begin{tabular}{ll}
\hline Ingredient Name & Diet \\
\hline Corn & 5.0 \\
\hline Wheat & 55.0 \\
Wheat bran & - \\
Rice bran & 13.54 \\
Alpha cellulose & - \\
\hline Soybean meal & 14.35 \\
\hline Canola ml solvent & 2.77 \\
Meat meal & 6.82 \\
Canola oil & 1.05 \\
\hline Limestone & - \\
\hline Dicalcium Phosphate & 0.5 \\
Phytase 5000 U/g & 0.01 \\
Salt & 0.12 \\
Sodium bicarbonate & 0.12 \\
UNE Vit conc & 0.05 \\
UNE TM conc & 0.08 \\
Choline Cl 60\% & 0.04 \\
L-lysine HCl 78.4 & 0.25 \\
\hline DL-methionine & 0.15 \\
L-threonine & 0.05 \\
Avizyme-1500 & 0.05 \\
Total & 100 \\
Formula cost (\$/Tonne) & 373.13 \\
Nutrients (\%) & \\
ME Poultry (Kcal/kg) & 3035.3 \\
ME Poultry(mj/kg) & 12.70 \\
Crude Protein & 21.0 \\
Crude Fiber & 3.01 \\
\hline Lysine & 1.19 \\
Methionine & 0.46 \\
Cystine & 0.33 \\
M+C & 0.80 \\
Calcium & 0.84 \\
Phosphorus avail & 0.76 \\
Phosphorus tot & 1.00 \\
\hline
\end{tabular}




\section{OPEN OACCESS}

\section{DiETARY TREATMENT}

The experiment design contained 6 dietary treatments (Table 1) with 6 replicates for each treatment. The chicks were fed on commercial types diets from 1-10 days (starter, Table 2). Subsequently, they were offered grower (11-24 days, Table 3) and finisher (25-35 days, Table 4) diets, which differed in fibre and energy content.

The diet ingredients for the birds in the starter phase are shown in Table 2. As broiler fat deposition occurred in the later phases, all of the birds were fed with the same diet formula. Tables 3 and 4 indicate the diet ingredients for the grower birds and the finisher birds, respectively. We focused on 4 typical diets that were used by the industry, formulated to contain only vegetable material or also containing animal by-products, and also supplemented with microbial enzymes (routinely done) or fed without such enzymes. The crude protein remained the same in all six diets. The diets 1 and 2 were the control diets with low fibre content. Diets 3-6 were the test diets. Diets 3 and 4 had optimum energy level and low energy level, respectively with medium fibre content. Diets 5 and 6 included high fibre content with optimum energy level and low energy level respectively. All of these diets and water were supplied without any restriction.

Table 3: Diet ingredients in the six dietary treatments for grower broilers (11-24 days).

\begin{tabular}{|c|c|c|c|c|c|c|}
\hline Ingredient name & Diet 1 & Diet 2 & Diet 3 & Diet 4 & Diet 5 & Diet 6 \\
\hline Corn & 5.0 & 22.1 & - & 2.0 & - & - \\
\hline Wheat & 70.8 & 50.7 & 60.0 & 60.0 & 51.5 & 49.2 \\
\hline Wheat bran & - & 0.99 & 0.67 & 2.0 & 4.0 & 6.6 \\
\hline Rice bran & 4.28 & 2 & 15 & 14.14 & 15.0 & 15.0 \\
\hline Alpha cellulose & - & 21.2 & 0.20 & 0.03 & 0.87 & 0.65 \\
\hline Soybean meal & 10.8 & 1.19 & 8.40 & 4.61 & 8.9 & 13.8 \\
\hline Canola ml solvent & - & 0.43 & 6.9 & 9.07 & 10.07 & 8.28 \\
\hline Meat meal & 6.57 & 0.02 & 4.26 & 5.42 & 2.77 & - \\
\hline Canola oil & 0.84 & 0.25 & 2.59 & 0.84 & 4.58 & 3.58 \\
\hline Limestone & 0.12 & 0.26 & 0.50 & 0.24 & 1.01 & 1.58 \\
\hline Dicalcium Phosphate & 0.5 & 0.05 & 0.5 & 0.5 & 0.05 & 0.05 \\
\hline Phytase $5000 \mathrm{U} / \mathrm{g}$ & 0.01 & 0.08 & 0.01 & 0.01 & 0.01 & 0.01 \\
\hline Salt & 0.02 & 0.01 & 0.01 & 0.12 & 0.12 & 0.10 \\
\hline Sodium bicarbonate & 0.10 & 0.27 & 0.02 & 0.10 & 0.10 & 0.10 \\
\hline UNE Vit conc & 0.05 & 0.17 & 0.05 & 0.05 & 0.05 & 0.05 \\
\hline UNE TM conc & 0.08 & 0.01 & 0.08 & 0.08 & 0.08 & 0.08 \\
\hline Choline $\mathrm{Cl} 60 \%$ & 0.04 & 0.05 & 0.05 & 0.06 & 0.06 & 0.06 \\
\hline L-lysine $\mathrm{HCl} 78.4$ & 0.41 & 0.37 & 0.40 & 0.44 & 0.39 & 0.35 \\
\hline DL-methionine & 0.18 & 0.17 & 0.17 & 0.17 & 0.17 & 0.17 \\
\hline L-threonine & 0.01 & 0.01 & 0.10 & 0.05 & 0.10 & 0.05 \\
\hline Avizyme-1500 & 0.05 & 0.05 & 0.05 & 0.05 & 0.05 & 0.05 \\
\hline Total & 100 & 100 & 100 & 100 & 100 & 100 \\
\hline Formula cost ( $\$ /$ Tonne) & 367.2 & 358.9 & 368.4 & 356.2 & 376.3 & 362.4 \\
\hline \multicolumn{7}{|l|}{ Nutrients (\%) } \\
\hline ME Poultry $(\mathrm{Kcal} / \mathrm{kg})$ & 3107.0 & 2987.5 & 3107.0 & 2987.5 & 3107.0 & 2987.5 \\
\hline ME Poultry $(\mathrm{mj} / \mathrm{kg})$ & 13 & 12.5 & 13 & 12.5 & 13 & 12.5 \\
\hline Crude Protein & 19.0 & 19.0 & 19.0 & 19.0 & 19.0 & 19.0 \\
\hline Crude Fiber & 2.5 & 2.5 & 3.5 & 3.5 & 4.5 & 4.5 \\
\hline Lysine & 1.15 & 1.12 & 1.17 & 1.19 & 1.19 & 1.18 \\
\hline Methionine & 0.45 & 0.45 & 0.46 & 0.46 & 0.46 & 0.46 \\
\hline Cystine & 0.30 & 0.30 & 0.32 & 0.33 & 0.33 & 0.34 \\
\hline $\mathrm{M}+\mathrm{C}$ & 0.76 & 0.76 & 0.79 & 0.80 & 0.81 & 0.81 \\
\hline Calcium & 0.84 & 0.84 & 0.84 & 0.84 & 0.84 & 0.84 \\
\hline Phosphorus avail & 0.61 & 0.57 & 0.69 & 0.73 & 0.57 & 0.49 \\
\hline Phosphorus tot & 0.79 & 0.57 & 0.95 & 1.00 & 0.85 & 0.78 \\
\hline
\end{tabular}


Table 4: Finisher (25-35 days) diets compositions in six diets.

\begin{tabular}{|c|c|c|c|c|c|c|}
\hline Ingredients & Diet 1 & Diet 2 & Diet 3 & Diet 4 & Diet 5 & Diet 6 \\
\hline Corn & 5.00 & 5.00 & 5.00 & 5.53 & 1.22 & - \\
\hline Wheat & 64.15 & 59.51 & 67.18 & 57.90 & 70.00 & 65.53 \\
\hline Wheat bran & - & 5.79 & - & - & - & - \\
\hline Rice bran & 14.72 & 15.00 & 7.42 & 15.00 & 10.19 & 11.22 \\
\hline Oat hulls & - & - & 1.00 & & 2.00 & - \\
\hline Alpha cellulose & 0.78 & 0.29 & 1.84 & 1.67 & 2.54 & 2.90 \\
\hline Soybean meal & 4.03 & 3.49 & 6.10 & 15.00 & & 13.39 \\
\hline Corn gluten & 2.50 & 2.00 & 0.94 & - & 4.00 & - \\
\hline Meat meal & 4.50 & 4.80 & 5.64 & - & 6.35 & 1.49 \\
\hline Canola oil & 2.11 & 1.91 & 3.05 & 2.46 & 2.50 & 3.04 \\
\hline Limestone & 0.66 & 0.58 & 0.50 & 1.52 & 0.17 & 0.96 \\
\hline Dicalcium Phosphate & - & - & 0.05 & 0.05 & 0.05 & 0.50 \\
\hline Phytase 5000 U/g & 0.01 & 0.01 & 0.01 & 0.01 & 0.01 & 0.01 \\
\hline Salt & 0.25 & 0.25 & 0.25 & 0.01 & 0.02 & 0.12 \\
\hline Na bicarbonate & 0.25 & 0.25 & 0.10 & 0.01 & 0.01 & 0.10 \\
\hline UNE Vit conc $(0.5 \mathrm{~kg} / \mathrm{mt})$ & 0.05 & 0.05 & 0.05 & 0.05 & 0.05 & 0.05 \\
\hline UNE TM conc $(0.75 \mathrm{~kg} / \mathrm{mt})$ & 0.08 & 0.08 & 0.08 & 0.08 & 0.08 & 0.08 \\
\hline Choline $\mathrm{Cl}$ 60\% & 0.07 & 0.08 & 0.02 & 0.05 & 0.01 & 0.04 \\
\hline L-lysine $\mathrm{HCl} 78.4$ & 0.54 & 0.57 & 0.45 & 0.35 & 0.58 & 0.35 \\
\hline DL-methionine & 0.16 & 0.18 & 0.17 & 0.17 & 0.15 & 0.17 \\
\hline L-threonine & 0.10 & 0.10 & 0.10 & 0.10 & 0.10 & 0.10 \\
\hline Avizyme 1500 & 0.05 & 0.05 & 0.05 & 0.05 & 0.05 & 0.05 \\
\hline Total & 100.0 & 100.0 & 100.0 & 100.0 & 100.0 & 100.0 \\
\hline Feed Cost (\$/Tonne): & 360.8 & 348.2 & 371.5 & 359.6 & 368.1 & 368.3 \\
\hline \multicolumn{7}{|l|}{ Nutrients (\%) } \\
\hline ME Poultry (kcal/kg) & 3202.6 & 3107.0 & 3202.6 & 3107.0 & 3202.6 & 3107.05 \\
\hline ME Poultry $(\mathrm{Mj} / \mathrm{kg})$ & 13.4 & 13 & 13.4 & 13 & 13.4 & 13 \\
\hline Crude Protein & 17 & 17 & 17 & 17 & 17 & 17 \\
\hline Crude fat & 6 & 6 & 6 & 5.9 & 6 & 6 \\
\hline Crude Fiber & 3.5 & 3.5 & 4.5 & 4.5 & 5.5 & 5.5 \\
\hline Lysine & 1.06 & 1.09 & 1.04 & 1.05 & 1.05 & 1.04 \\
\hline Methionine & 0.42 & 0.43 & 0.42 & 0.41 & 0.42 & 0.41 \\
\hline Cystine & 0.28 & 0.28 & 0.27 & 0.28 & 0.28 & 0.28 \\
\hline d Lys pou & 0.95 & 0.95 & 0.91 & 0.95 & 0.92 & 0.93 \\
\hline d Met pou & 0.39 & 0.39 & 0.39 & 0.39 & 0.39 & 0.39 \\
\hline Calcium & 0.76 & 0.76 & 0.821 & 0.76 & 0.76 & 0.76 \\
\hline Phosphorus total & 0.79 & 0.85 & 0.72 & 0.66 & 0.80 & 0.72 \\
\hline
\end{tabular}

SAMPLing

At day 10, one bird from each cage was slaughtered by cervical dislocation, and they were stored in a freezer. At day 24 , two birds were selected from each cage and slaughtered by cervical dislocation. Their visceral organs were weighed, and the digesta from the ileum and caeca were obtained and pooled. These samples were freeze-dried and used to measure gross energy and crude protein, as well as starch contents for the estimation of digestibility. Digesta was also collected from the jejunum for viscosity analysis. At day 35, 2 birds from each cage were slaughtered by cervical dislocation. The organs and digesta were collected and measured as done at day 24. Then, the breast meat and abdominal fat pad were collected for meat yield test and fat analysis. 


\section{STATISTICAL ANALYSIS}

Data were subjected to the analysis of variance using the General Linear Model (GLM) of the IBM SPSS Statistics software Version 20. One-way analysis of variance (ANOVA) was carried out using the following model:

$$
\mathrm{y}_{i j k}=\mathrm{u}+\mathrm{A}_{i}+\mathrm{B}_{j}+(\mathrm{AB})_{i j}+\mathrm{e}_{i j k}
$$

Which;

$\mathrm{y}_{i j k}$ is the observation $k$ in level $i$ of dietary energy levels and level $j$ of fibre contents in the diets $\mathrm{u}$ is the overall mean; $\mathrm{A}_{i}$ is the effect of level $i$ of energy levels, $i=1,2 ; i=$ $1=$ optimum dietary energy level and $i=2=$ low dietary energy level; $\mathrm{B}_{j}$ is the effect of level $\mathrm{j}$ of fibre contents in the diets, $j=1,2,3 ; j=1$ = low fibre content, $j=2$ = medium fibre content and $j=3=$ high fibre content; $(\mathrm{AB})_{i j}$ is the effect of the interaction of level $i$ of dietary energy levels with level $j$ of fibre contents in the diets; eijk is the random error mean 0 and variance $\delta^{2}$.

Separation of means within a significant effect was done by Duncan's Multiple Range Test (DMRT) through post hoc procedure of SPSS. Differences were considered significant at $\mathrm{P}<0.05$. The results were expressed as means with their standard errors of the mean.

\section{RESULTS AND DISCUSSION}

\section{FEED INTAKE, BODY WEIGHT AND FEED CONVERSION RATIO (FCR)}

As Table 5 shown, feed intake was not significantly $(\mathrm{P}$ > 0.098) impacted by the fibre contents in the diet. However, the birds with low fibre content diet gained higher $(\mathrm{P}<$ 0.011) body weight than birds with other two fibre content diets. Consequently, birds fed on low fibre content diet had higher body weight than birds fed on higher fibre content diets. The body weights of birds were not significantly impacted by medium dietary fibre and high dietary fibre. Thus, the diet with high fibre level had significantly higher FCR $(\mathrm{P}<0.001)$ than the other two diets.

Feed intake was significantly affected by the energy level. The birds took more feed $(\mathrm{P}=0.98)$ with optimum energy level than feed with low energy level. Nevertheless, the birds with optimum energy level gained more body weight $(\mathrm{P}<0.011)$ than the birds with low energy level. For the FCR, there was not predominantly different between the two diets.

\section{VISCERAL ORGAN WEIGHTS}

As Table 6 shown, seven types of visceral organs were measured. The energy level did not have any significant impact on visceral organs, but the fibre content did affect some of them. The relative weight of the small intestine was significantly larger $(\mathrm{P}<0.019)$ on the high fibre diet than a low fibre diet. However, small intestinal weight was not significantly different between birds with low fibre content diet and medium fibre content diet, and between birds with high fibre content diet and medium fibre content diet. Meanwhile, the fibre content had the same impact on liver weight. The liver weight was higher $(\mathrm{P}<0.056)$ on the high fibre diet than a low fibre diet, but no difference between high (or low) fibre content diet and medium fibre content diet. The weight of the other visceral organs was not impacted by dietary fibre content.

Table 5: Feed intake, body weight, body weight gain and FCR of broiler chickens reared on different grower and finisher diets from 11 to 35 days of age.

\begin{tabular}{|c|c|c|c|c|c|}
\hline \multicolumn{2}{|l|}{ Treatments } & \multirow[t]{2}{*}{$\begin{array}{l}\text { Feed } \\
\text { intake } \\
(\mathrm{g} / \mathrm{b})\end{array}$} & \multirow[t]{2}{*}{$\begin{array}{l}\text { Body } \\
\text { weight } \\
\text { (g/b) }\end{array}$} & \multirow[t]{2}{*}{$\begin{array}{l}\text { Body } \\
\text { weight } \\
\text { gain }(g / b)\end{array}$} & \multirow[t]{2}{*}{ FCR } \\
\hline Fibre contents & $\begin{array}{l}\text { Energy } \\
\text { levels }\end{array}$ & & & & \\
\hline Low & Optimum & 3432.0 & 2250.9 & 2209.6 & 1.55 \\
\hline Low & Low & 3248.0 & 2177.2 & 2136.1 & 1.52 \\
\hline Medium & Optimum & 3332.9 & 2143.6 & 2102.2 & 1.59 \\
\hline Medium & Low & 3337.7 & 2026.3 & 1984.7 & 1.68 \\
\hline High & Optimum & 3510.5 & 2142.9 & 2101.7 & 1.67 \\
\hline High & Low & 3324.7 & 2103.8 & 2062.8 & 1.61 \\
\hline SEM & & 28.2 & 18.8 & 18.8 & 0.014 \\
\hline Model P value & & 0.098 & $<0.011$ & $<0.011$ & $<0.001$ \\
\hline \multicolumn{6}{|l|}{ Main effects } \\
\hline Low & & 3340.0 & $2214.0^{a}$ & $2172.9^{a}$ & $1.54^{\mathrm{b}}$ \\
\hline Medium & & 3335.3 & $2084.9^{\mathrm{b}}$ & $2043.4^{\mathrm{b}}$ & $1.63^{\mathrm{b}}$ \\
\hline \multirow[t]{3}{*}{ High } & & 3417.6 & $2123.3^{b}$ & $2082.2^{\mathrm{b}}$ & $1.64^{\mathrm{a}}$ \\
\hline & Low & $3303.5^{b}$ & $2102.4^{\mathrm{b}}$ & $2061.2^{\mathrm{b}}$ & 1.61 \\
\hline & Optimum & $3425.2^{a}$ & $2179.1^{\mathrm{a}}$ & $2137.8^{\mathrm{a}}$ & 1.60 \\
\hline \multicolumn{6}{|c|}{ Interactions terms } \\
\hline Crude fiber leve & & NS & $<0.008$ & $<0.008$ & $<0.000$ \\
\hline Energy level & & $<0.028$ & $<0.023$ & $<0.023$ & NS \\
\hline $\begin{array}{l}\text { Crude fiber X e } \\
\text { level }\end{array}$ & energy & NS & NS & NS & $<0.011$ \\
\hline
\end{tabular}

\section{MEAT YIELD CHARACTERISTICS}

The dietary energy level did not have a significant effect on the carcass characteristics except for the abdominal fat (Table 7). The birds with optimum dietary energy level produced larger $(\mathrm{P}<0.000)$ abdominal fat pad.

The dietary fibre also had significant $(\mathrm{P}<0.000)$ impact on the abdominal fat. The birds with medium (and high) fibre content diet deposited less abdominal fat than birds with low fibre content diet. Meanwhile, breast yield was significantly higher $(\mathrm{P}<0.027)$ on high fibre content diet than other two diets. Breast yield did not have significant differences between birds fed on low fibre content diet and birds fed on medium fibre content diet. The other characteristics were not affected by dietary fibre content. 
Table 6: Visceral organ weights ( $\mathrm{g} / 100 \mathrm{~g}$ of body weight) of broiler chicken at 35 days of age

\begin{tabular}{|c|c|c|c|c|c|c|c|c|}
\hline \multicolumn{2}{|l|}{ Treatments } & \multirow[t]{2}{*}{ Small intest ${ }^{+}$} & \multirow{2}{*}{\multicolumn{2}{|c|}{$\begin{array}{l}\text { Proventricu- Gizzard } \\
\text { lus }^{+}\end{array}$}} & \multirow[t]{2}{*}{ Pancr-ease } & \multirow[t]{2}{*}{ Spleen } & \multirow[t]{2}{*}{ Liver } & \multirow[t]{2}{*}{ Bursa } \\
\hline Fiber & Energy & & & & & & & \\
\hline Low & Optimum & 2.2 & 0.4 & 1.1 & 0.2 & 0.1 & 2.0 & 0.2 \\
\hline Low & Low & 2.4 & 0.4 & 1.0 & 0.2 & 0.1 & 2.1 & 0.2 \\
\hline Medium & Optimum & 2.4 & 0.4 & 0.8 & 0.2 & 0.1 & 2.1 & 0.2 \\
\hline Medium & Low & 2.6 & 0.4 & 1.1 & 0.2 & 0.1 & 2.3 & 0.2 \\
\hline High & Optimum & 2.7 & 0.4 & 1.0 & 0.2 & 0.1 & 2.3 & 0.2 \\
\hline High & Low & 2.6 & 0.4 & 0.9 & 0.2 & 0.1 & 2.4 & 0.2 \\
\hline SEM & & 0.0 & 0.0 & 0.0 & 0.0 & 0.0 & 0.0 & 0.0 \\
\hline $\mathrm{P}$ value & & $<0.019$ & 0.807 & 0.6 & 0.779 & 0.861 & $<0.056$ & 0.5 \\
\hline \multicolumn{9}{|l|}{ Main effects } \\
\hline Low & & $2.28^{\mathrm{b}}$ & 0.4 & 1.1 & 0.2 & 0.1 & $2.07^{\mathrm{b}}$ & 0.2 \\
\hline Medium & & $2.48^{\mathrm{ab}}$ & 0.4 & 1.0 & 0.2 & 0.1 & $2.16^{\mathrm{ab}}$ & 0.2 \\
\hline \multirow[t]{3}{*}{ High } & & $2.63^{\mathrm{a}}$ & 0.4 & 1.0 & 0.2 & 0.1 & $2.36^{a}$ & 0.2 \\
\hline & Low & 2.5 & 0.4 & 1.0 & 0.2 & 0.1 & 2.3 & 0.2 \\
\hline & Optimum & 2.4 & 0.4 & 1.0 & 0.2 & 0.1 & 2.1 & 0.2 \\
\hline \multicolumn{9}{|c|}{ Interactions terms } \\
\hline \multicolumn{2}{|c|}{ Crude fiber level } & $<0.006$ & 0.6 & 0.7 & 0.3 & 0.5 & $<0.02$ & 0.4 \\
\hline \multicolumn{2}{|c|}{ Energy level } & 0.3 & 0.8 & 0.9 & 0.8 & 0.5 & 0.1 & 0.4 \\
\hline \multicolumn{2}{|c|}{ Crude fiber X energy } & 0.2 & 0.6 & 0.2 & 0.9 & 1.0 & 1.0 & 0.3 \\
\hline
\end{tabular}

${ }^{+}$Small intestine, proventriculus and gizzard considered without digesta content. ${ }^{\text {a-b }}$ Different superscripts in the same column differ significantly.

Table 7: Meat yield characteristics (g/bird) of broiler chickens fed different types of grower and finisher diets at 35 days of age.

\begin{tabular}{|c|c|c|c|c|c|c|c|c|}
\hline \multicolumn{2}{|c|}{ Treatments } & \multirow[t]{2}{*}{ Live weight } & \multirow[t]{2}{*}{$\begin{array}{l}\text { Carcass } \\
\text { weight }\end{array}$} & \multirow[t]{2}{*}{ Thigh } & \multirow[t]{2}{*}{ Drumstick } & \multirow[t]{2}{*}{$\begin{array}{l}\text { Breast (with- } \\
\text { out skin) }\end{array}$} & \multirow[t]{2}{*}{ Wings } & \multirow[t]{2}{*}{$\begin{array}{l}\text { Abdominal } \\
\text { fat pad }\end{array}$} \\
\hline Fiber & Energy & & & & & & & \\
\hline Low & Optimum & 2248.4 & 1678.3 & 263.9 & 216.4 & 416.5 & 170.4 & 30.6 \\
\hline Low & Low & 2201.0 & 1630.0 & 250.1 & 215.5 & 392.2 & 163.7 & 23.0 \\
\hline Medium & Optimum & 2179.2 & 1629.3 & 250.9 & 213.5 & 395.0 & 159.2 & 25.1 \\
\hline Medium & Low & 2093.2 & 1562.3 & 240.4 & 199.9 & 391.8 & 157.4 & 22.6 \\
\hline High & Optimum & 2201.2 & 1621.2 & 244.2 & 207.5 & 413.4 & 158.5 & 22.2 \\
\hline High & Low & 2250.6 & 1684.0 & 278.3 & 211.2 & 410.9 & 168.5 & 24.6 \\
\hline SEM & & 19.56 & 16.71 & 6.52 & 2.31 & 6.81 & 1.84 & 1.039 \\
\hline$P$ value & & 0.200 & 0.330 & 0.594 & 0.319 & $<0.027$ & 0.183 & $<0.000$ \\
\hline \multicolumn{9}{|c|}{ Main effects } \\
\hline Low & & 2224.7 & 1654.2 & 257.0 & 216.0 & $407.6^{\mathrm{b}}$ & 167.1 & $28.2^{\mathrm{a}}$ \\
\hline Medium & & 2136.2 & 1595.8 & 245.6 & 206.7 & $397.6^{\mathrm{b}}$ & 158.3 & $22.5^{\mathrm{b}}$ \\
\hline \multirow[t]{3}{*}{ High } & & 2225.9 & 1652.6 & 261.2 & 209.3 & $430.1^{a}$ & 163.5 & $23.7^{\mathrm{b}}$ \\
\hline & Low & 2181.6 & 1625.4 & 256.2 & 208.8 & 409.1 & 163.2 & $22.6^{\mathrm{b}}$ \\
\hline & Optimum & 2209.6 & 1642.9 & 253.0 & 212.5 & 414.4 & 162.7 & $27.0^{\mathrm{a}}$ \\
\hline \multicolumn{9}{|c|}{ Interactions terms } \\
\hline \multicolumn{2}{|c|}{ Crude fiber level } & 0.100 & 0.272 & 0.616 & 0.248 & $<0.010$ & 0.141 & $<0.002$ \\
\hline \multicolumn{2}{|c|}{ Energy level } & 0.462 & 0.599 & 0.809 & 0.434 & 0.528 & 0.896 & $<0.002$ \\
\hline \multicolumn{2}{|c|}{ Crude fiber X energy } & NS & NS & NS & NS & 0.175 & 0.157 & $<0.019$ \\
\hline
\end{tabular}

${ }^{a-b}$ Different superscripts in the same column differ significantly. 
Table 8: Economic feed cost analysis (AU\$), according to the Australian current feed ingredient price.

\begin{tabular}{|c|c|c|c|c|c|c|c|c|c|c|c|c|}
\hline \multicolumn{2}{|c|}{ Treatments } & \multicolumn{3}{|c|}{ Starter (d0-10) } & \multicolumn{3}{|c|}{ Grower (d11-24) } & \multicolumn{3}{|c|}{ Finisher (d25-350) } & \multicolumn{2}{|c|}{ Overall (0d-35) } \\
\hline Fiber & Energy & $\begin{array}{l}\text { Formula } \\
\text { cost }(\$ / \\
\text { kg feed }\end{array}$ & $\begin{array}{l}\text { Feed } \\
\text { cost }(\$ / \\
\text { bird) }\end{array}$ & $\begin{array}{l}\text { Feed } \\
\text { cost }(\$ / \\
\operatorname{kg} \text { gain })\end{array}$ & $\begin{array}{l}\text { Formula } \\
\text { cost }(\$ / \\
\mathrm{kg} \text { feed }\end{array}$ & $\begin{array}{l}\text { Feed } \\
\text { cost }(\$ / \\
\text { bird) }\end{array}$ & $\begin{array}{l}\text { Feed } \\
\text { cost }(\$ / \\
\text { kg gain) }\end{array}$ & $\begin{array}{l}\text { Formula } \\
\text { cost }(\$ / \\
\mathrm{kg} \text { feed }\end{array}$ & $\begin{array}{l}\text { Feed } \\
\text { cost }(\$ / \\
\text { bird) }\end{array}$ & $\begin{array}{l}\text { Feed } \\
\text { cost (\$/ } \\
\text { kg gain) }\end{array}$ & $\begin{array}{l}\text { Feed } \\
\text { cost }(\$ / \\
\text { bird })\end{array}$ & $\begin{array}{l}\text { Feed } \\
\text { cost }(\$ / \\
\text { kg gain })\end{array}$ \\
\hline Low & Optimum & 0.37 & 0.11 & 0.45 & 0.37 & 0.60 & 0.57 & 0.36 & 0.54 & 0.60 & 1.25 & 0.57 \\
\hline Low & Low & 0.37 & 0.10 & 0.45 & 0.36 & 0.53 & 0.53 & 0.35 & 0.52 & 0.58 & 1.16 & 0.54 \\
\hline Medium & Optimum & 0.37 & 0.11 & 0.45 & 0.37 & 0.55 & 0.58 & 0.37 & 0.58 & 0.62 & 1.23 & 0.58 \\
\hline Medium & Low & 0.37 & 0.11 & 0.45 & 0.36 & 0.55 & 0.56 & 0.36 & 0.53 & 0.70 & 1.19 & 0.60 \\
\hline High & Optimum & 0.37 & 0.10 & 0.45 & 0.38 & 0.58 & 0.57 & 0.36 & 0.61 & 0.72 & 1.30 & 0.62 \\
\hline High & Low & 0.37 & 0.10 & 0.44 & 0.36 & 0.53 & 0.56 & 0.36 & 0.58 & 0.65 & 1.21 & 0.59 \\
\hline SEM & & 0.000 & 0.001 & 0.002 & 0.001 & 0.007 & 0.004 & 0.001 & 0.008 & 0.011 & 0.012 & 0.005 \\
\hline $\mathrm{P}$ value & & NS & NS & NS & NS & $<0.00$ & $<0.00$ & NS & $<0.00$ & $<0.00$ & $<0.00$ & $<0.00$ \\
\hline \multicolumn{13}{|c|}{ Main effects } \\
\hline Low & & 0.37 & 0.11 & 0.45 & 0.36 & 0.57 & $0.55^{\mathrm{b}}$ & 0.35 & $0.53^{\mathrm{b}}$ & $0.59^{\mathrm{b}}$ & $1.20^{\mathrm{b}}$ & $0.55^{\mathrm{b}}$ \\
\hline Medium & & 0.37 & 0.11 & 0.45 & 0.36 & 0.55 & $0.57^{\mathrm{a}}$ & 0.36 & $0.55^{\mathrm{b}}$ & $0.66^{\mathrm{a}}$ & $1.21^{\mathrm{ab}}$ & $0.59^{\mathrm{a}}$ \\
\hline \multirow[t]{3}{*}{ High } & & 0.37 & 0.10 & 0.45 & 0.37 & 0.55 & $0.57^{\mathrm{a}}$ & 0.36 & $0.59^{\mathrm{a}}$ & $0.68^{\mathrm{a}}$ & $1.25^{\mathrm{a}}$ & $0.60^{\mathrm{a}}$ \\
\hline & Low & 0.37 & 0.11 & 0.45 & 0.36 & $0.54^{\mathrm{b}}$ & $0.55^{\mathrm{b}}$ & 0.36 & $0.54^{\mathrm{b}}$ & 0.64 & $1.19^{\mathrm{b}}$ & 0.58 \\
\hline & Optimum & 0.37 & 0.11 & 0.45 & 0.37 & $0.58^{\mathrm{a}}$ & $0.57^{\mathrm{a}}$ & 0.36 & $0.57^{\mathrm{a}}$ & 0.65 & $1.26^{\mathrm{a}}$ & 0.59 \\
\hline \multicolumn{13}{|c|}{ Interactions terms } \\
\hline \multicolumn{2}{|c|}{ Crude fiber level } & NS & NS & NS & NS & NS & $<0.00$ & NS & $<0.00$ & $<0.00$ & $<0.00$ & $<0.00$ \\
\hline \multicolumn{2}{|c|}{ Energy level } & NS & NS & NS & NS & $<0.00$ & $<0.00$ & NS & $<0.01$ & NS & $<0.00$ & 0.098 \\
\hline \multicolumn{2}{|c|}{ Crude fiber X energy } & NS & NS & NS & NS & $<0.01$ & NS & NS & NS & $<0.00$ & NS & $<0.02$ \\
\hline
\end{tabular}

${ }^{a-b}$ Different superscripts in the same column differ significantly.

\section{ECONOMIC ANALYSIS}

According to Table 8, the feed cost was the same because of the same diet for all cages. On the grower phase, the fibre content had no significant effect on feed cost on birds (\$/ bird). However, the feed cost on meat gained was higher ( $\mathrm{P}$ $<0.00$ ) on high fibre content diet than a low fibre content diet. In the meantime, the feed cost, either on birds or on meat gain, was significantly higher $(\mathrm{P}<0.00)$ on the high (optimum) energy level than low energy level diet. For the finisher birds, both feed costs were significantly higher $(\mathrm{P}<0.00)$ on the high fibre content diet than a low fibre content diet. Feed cost $(\$ /$ birds) was higher $(\mathrm{P}<0.00)$ on high energy level diet than low energy level diet, whilst feed cost ( $\$ / \mathrm{kg}$ gain) was not significantly impacted by the dietary energy level.

The overall feed cost on birds (\$/bird) was significantly higher $(\mathrm{P}<0.00)$ with high fibre content diet than a low fibre content diet. The medium fibre content would have no significant difference with high fibre content or low fibre content diet. Meanwhile, the total feed cost on meat gain $(\$ / \mathrm{kg}$ gain) would be higher $(\mathrm{P}<0.00)$ with high fibre content diet than low fibre content diet, but no significant difference with medium fibre content diet. gain) was not significantly impacted by the energy level in diets. However, the total feed cost on birds (\$/bird) is significantly affected. The diet with optimum dietary energy cost more $(\mathrm{P}<0.00)$ than the low dietary energy diet.

From the report, feed intake was not significantly $(\mathrm{P}>$ 0.098) impacted by the fibre contents in the diet, which is in agreement with Kras et al. (2013) who suggested that feed intake was not impacted by dietary fibre level. However, the birds gain significantly more body weights on a low fibre content diet than on diet with higher fibre content. Kras et al. (2013) also figured out that broilers fed on high fibre diets had worse performance. The body weights of birds were not significantly impacted by medium dietary fibre and high dietary fibre, which is in agreement with Jorgensen et al. (1996). Jorgensen et al. (1996) suggested that body weight gain did not differ significantly between the medium and high dietary fibre contents. The birds fed on high fibre content intake no significantly different feed but gained lower body weight, which indicated a higher FCR for high fibre content diets. This finding matched data of Kras et al. (2013), indicated the birds fed a high fibre content diet presented worse FCR.

For dietary energy, the total feed cost on meat gain $(\$ / \mathrm{kg}$

Birds fed on high fibre content diet generated larger 
small intestine. This is in agreement with previous studies (Moss, 1989; Savory, 1992; Onifade, 1997). The authors of previous studies (Moss, 1989; Savory, 1992; Onifade, 1997) commented that dietary fibre could impact the length and weight of the GI tract, and both the weight and length of the GI tract increased with increasing dietary level, especially the caeca increased considerably. Larger small intestine indicated more microbes which in turns to higher digestibility (Hubener et al., 2002). The NSP fraction can, to a certain degree, be broken down by the microbial flora permanently colonising the gastrointestinal (GI) tract (Jorgensen et al., 1996). By the means of dietary fibre degradation, the microflora may benefit host bird by supplying extra energy, and the benefit would be great if energy supply was insufficient. This study also found that birds had larger liver with high fibre content diet than birds with low fibre content diet, whereas Mohiti-Asli et al. (2012) indicated a reduced liver weight with the increase of dietary fibre content. Mohiti-Asli et al. (2012) suggested that high dietary fibre could depress lipid accumulation in the liver, thus the weight of the liver would be reduced.

Broilers can produce more breast meat on high fibre content diet than the lower fibre content diets. Onifade et al. (1999) reported that birds gained more breast weights with high fibre diets. From Table 7, the carcass weight was not significantly influenced by the dietary fibre content. The carcass had no significant difference among birds fed on high, medium and low fibre content diets, but the breast meat yielded highest on the high dietary fibre content. Meanwhile, the other parts of the birds also did not have a significant impact on the dietary fibre content. Thus, it is because the birds with low dietary fibre content deposited more fat than birds with high fibre content diet. Broilers fed on high fibre content diet deposited less abdominal fat. This finding is in agreement with data of Mohiti-Asli et al. (2012) and Kras et al. (2013). They commented that high fibre content in diet could reduce the abdominal fat deposition.

According to the study, birds consumed more diet with optimum energy level than a diet with a lower energy level. This finding matched the data of Lopez and Leeson (2008) and Azizi et al. (2011) who said broilers would intake more feed if the dietary energy is increased. Under the condition of ad libitum feeding, chickens can grow more rapidly with higher energy level (Khantaprab et al., 1997), especially in the later phases fat deposition would be fast with excess dietary energy (Leenstra, 1986). Thus, as stated, the body weight and body weight gain were higher on the optimum dietary energy level than on a low dietary energy level. However, the FCR was not significantly affected by the dietary energy level. Azizi et al. (2011) also stated that there were no significant differences were observed for FCR between birds fed high and low energy level from 1 to 42 days of age.

The weights of visceral organs were not significantly impacted by the dietary energy level, whereas in contrary to Jorgensen et al. (1996). They reported that there was a strong indication that differences in the weight of visceral organs are highly related to differences in energy consumption. The weights of visceral organs will increase with the increase of dietary energy because of the accumulation of the lipids.

The increase of dietary energy increased the body weight and body weight gain of broilers. However, comparison with the meat yield characteristics of birds on low dietary energy, the increase of dietary energy increased nothing except for abdominal fat. McLeod (1982) and Khantaprab et al. (1997) reported that birds with higher dietary energy would produce more abdominal fat.

The overall feed cost both on birds (\$/bird) and on meat gain ( $\$ / \mathrm{kg}$ gain) was higher on high fibre content diet than low fibre content diet. There were no significant differences between both feed cost in feed with high fibre content and feed with medium fibre content.

Feed cost, either on birds or on meat gain, was significantly higher $(\mathrm{P}<0.00)$ on the high (optimum) energy level than low energy level diet. In the grower phases, feed cost was significantly higher on the optimum energy level than on low energy level. For the finisher birds and from the overall perspective, feed costs on birds were significantly higher on the optimum energy level diet than low energy level diet. Whilst feed costs ( $\$ / \mathrm{kg}$ gain) were not significantly impacted by the dietary energy level. That is because there was much energy converted into fat.

\section{CONCLUSIONS AND RECOMMENDATIONS}

In conclusion, a high fibre content diet can produce more breast meat and less abdominal fat. However, the diet with high fibre level had significantly higher FCR than the other two diets, which means that the diet with high fibre content has lower economic efficiency. The feed cost of high fibre content diet will cost more than lower fibre diets. A low energy level diet can reduce feed intake without any particular impact on visceral organs development, reduce feed cost per bird and generate less abdominal fat. Nevertheless, lower dietary energy level made birds smaller.

\section{ACKNOWLEDGEMENTS}

The authors are grateful to the University of New England 
(UNE) for supporting the study providing financial supports through the University Research Seed Grants of UNE.

\section{AUTHOR'S CONTRIBUTION}

All authors contributted equally.

\section{CONFLICT OF INTEREST}

The authors have declared no conflict of interests.

\section{REFERENCES}

-Azizi B, Sadeghi G, Karimi A, Abed F (2011). Effects of dietary energy and protein dilution and time of feed replacement from starter to grower on broiler chickens performance. J. Cent. Eur. Agric., 12(1): 44-52. https://doi.org/10.5513/ JCEA01/12.1.879

- Da Costa MJ, Colson G, Frost TJ, Halley J, Pesti GM (2017). Straight-run vs. sex separate rearing for two broiler genetic lines Part 2: Economic analysis and processing advantages. Poult. Sci., 96(7): 2127-2136. https://doi.org/10.3382/ps/ pew498

-Donohue M, Cunningham DL (2009). Effects of grain and oilseed prices on the costs of US poultry production. J. Appl. Poult. Res., 18(2): 325-337. https://doi.org/10.3382/ japr.2008-00134

- Dozier III WA, Behnke KC, Gehring CK, Branton SL (2010). Effects of feed form on growth performance and processing yields of broiler chickens during a 42-day production period. J.Appl.Poult. Res., 19(3):219-226.https://doi.org/10.3382/ japr.2010-00156

- Ferket PR, Gernat AG (2006). Factors that affect feed intake of meat birds: A review. Int. J. Poult. Sci., 5(10): 905-911. https://doi.org/10.3923/ijps.2006.905.911

- González-Alvarado JM, Jiménez-Moreno E, Lázaro R, Mateos GG (2007). Effect of type of cereal, heat processing of the cereal, and inclusion of fiber in the diet on productive performance and digestive traits of broilers. Poult. Sci., 86(8): 1705-1715. https://doi.org/10.1093/ps/86.8.1705

- Griffiths L, Leeson S, Summers JD (1977). Fat deposition in broilers: Effect of dietary energy to protein balance, and early life caloric restriction on productive performance and abdominal fat pad size. Poult. Sci. 56(2): 638-646. https:// doi.org/10.3382/ps.0560638

-Hübener K, Vahjen W, Simon O (2002). Bacterial responses to different dietary cereal types and xylanase supplementation in the intestine of broiler chicken. Arch. Anim. Nutr., 56(3): 167-187. https://doi.org/10.1080/00039420214191

-Jørgensen H, Zhao XQ, Knudsen KE, Eggum BO (1996). The influence of dietary fibre source and level on the development of the gastrointestinal tract, digestibility and energy metabolism in broiler chickens. Br. J. Nutr., 75(3): 379-395. https://doi.org/10.1079/BJN19960141

-Khantaprab S, Nikki T, Nobukuni K (1997). Effect of restricted feed intake on the growth of muscle and the fat deposition in broiler chickens. Japanese Poult. Sci., 34(6): 363-372. https://doi.org/10.2141/jpsa.34.363

-Krás RV, Kessler AD, Ribeiro AM, Henn JD, Dos Santos II, Halfen DP, Bockor L (2013). Effect of dietary fiber and genetic strain on the performance and energy balance of broiler chickens. Braz. J. Poult. Sci., 15(1): 15-19. https:// doi.org/10.1590/S1516-635X2013000100003

-Leenstra FR (1986). Effect of age, sex, genotype and environment on fat deposition in broiler chickens. A review. Worlds. Poult. Sci. J., 42(1): 12-25. https://doi.org/10.1079/ WPS19860002

-Lippens M, Room G, De Groote G, Decuypere E (2000). Early and temporary quantitative food restriction of broiler chickens. 1. Effects on performance characteristics, mortality and meat quality. Br. Poult. Sci., 41(3): 343-354. https://doi. org/10.1080/713654926

- Lopez G, Leeson S (2008). Energy partitioning in broiler chickens. Can. J. Anim. Sci., 88(2): 2005-2012. https://doi. org/10.4141/CJAS07087

- Mabray CJ, Waldroup PW (1981). The influence of dietary energy and amino acid levels on abdominal fat pad development of the broiler chicken. Poult. Sci., 60(1): 151159. https://doi.org/10.3382/ps.0600151

- Mateos GG, Jiménez-Moreno E, Serrano MP, Lázaro RP (2012). Poultry response to high levels of dietary fiber sources varying in physical and chemical characteristics. J. Appl. Poult. Res., 21(1): 156-174. https://doi.org/10.3382/ japr.2011-00477

- Mcleod JA (1982). Nutritional factors influencing carcase fat in broilers. A review. World's. Poult. Sci. J., 38(3): 194-200. https://doi.org/10.1079/WPS19820015

- Mohiti-Asli M, Shivazad M,Zaghari M, Aminzadeh S, Rezaian M, Mateos GG (2012). Dietary fibers and crude protein content alleviate hepatic fat deposition and obesity in broiler breeder hens. Poult. Sci., 91(12): 3107-3114. https://doi. org/10.3382/ps.2011-02040

- Moss R (1989). Gut size and the digestion of fibrous diets by tetraonid birds. J. Exp. Zool., 252(S3): 61-65. https://doi. org/10.1002/jez.1402520510

- Onifade AA (1997). Growth performance, carcass characteristics, organs measurement and haematology of broiler chickens fed a high fibre diet supplemented with antibiotics or dried yeast. Food/Nahrung. 41(6): 370-374. https://doi. org/10.1002/food.19970410612

- Onifade AA, Odunsi AA, Babatunde GM, Olorede BR, Muma E (1999). Comparison of the supplemental effects of Saccharomyces cerevisiae and antibiotics in low-protein and high-fibre diets fed to broiler chickens. Arch. Anim. Nutr., 52(1): 29-39. https://doi.org/10.1080/17450399909386149

- Parkhurst CR, Mountney GJ (1988). Poultry and Egg Marketing. In: Poultry Meat and Egg Production. Springer, Boston, MA. https://doi.org/10.1007/978-1-4757-0683-3

- Saleh AA, El-Far AH, Abdel-Latif MA, Emam MA, Ghanem R, Abd El-Hamid HS (2018). Exogenous dietary enzyme formulations improve growth performance of broiler chickens fed a low-energy diet targeting the intestinal nutrient transporter genes. PLoS One, 13(5): e0198085. https://doi.org/10.1371/journal.pone.0198085

- Savory CJ (1992). Gastrointestinal morphology and absorption of monosaccharides in fowls conditioned to different types and levels of dietary fibre. Br. J. Nutr., 67(1): 77-89. https:// doi.org/10.1079/BJN19920010

-Zhou Y, Jiang Z, Lv D, Wang T (2009). Improved energyutilizing efficiency by enzyme preparation supplement in broiler diets with different metabolizable energy levels. Poult. Sci., 88(2): 316-322. https://doi.org/10.3382/ ps.2008-00231 